\title{
Article \\ Correlation of an Index-Lesion-Based SPECT Dosimetry Method with Mean Tumor Dose and Clinical Outcome after ${ }^{177}$ Lu-PSMA-617 Radioligand Therapy
}

\author{
Friederike Völter ${ }^{1}{ }^{\circledR}$, Lena Mittlmeier ${ }^{1}$, Astrid Gosewisch ${ }^{1}$, Julia Brosch-Lenz ${ }^{1}$, Franz Josef Gildehaus ${ }^{1}$, \\ Mathias Johannes Zacherl ${ }^{1}$, Leonie Beyer ${ }^{1}{ }^{\mathbb{D}}$, Christian G. Stief ${ }^{2}$, Adrien Holzgreve ${ }^{1}$, Johannes Rübenthaler ${ }^{3}{ }^{\circ}$, \\ Clemens C. Cyran ${ }^{3} \mathbb{D}$, Guido Böning ${ }^{1}$, Peter Bartenstein ${ }^{1}$, Andrei Todica ${ }^{1} \mathbb{D}$ and Harun Ilhan ${ }^{1, *}$ \\ check for \\ updates \\ Citation: Völter, F.; Mittlmeier, L.; \\ 1 Department of Nuclear Medicine, University Hospital, Ludwig-Maximilians-University Munich, \\ 80331 Munich, Germany; friederike.voelter@med.uni-muenchen.de (F.V.); \\ lena.mittlmeier@med.uni-muenchen.de (L.M.); astrid.gosewisch@med.uni-muenchen.de (A.G.); \\ julia.brosch-lenz@med.uni-muenchen.de (J.B.-L.); franz.gildehaus@med.uni-muenchen.de (F.J.G.); \\ mathias.zacherl@med.uni-muenchen.de (M.J.Z.); leonie.beyer@med.uni-muenchen.de (L.B.); \\ adrien.holzgreve@med.uni-muenchen.de (A.H.); guido.boening@med.uni-muenchen.de (G.B.); \\ peter.bartenstein@med.uni-muenchen.de (P.B.); andrei.todica@med.uni-muenchen.de (A.T.) \\ 2 Department of Urology, University Hospital, Ludwig-Maximilians-University Munich, 80331 Munich, \\ Germany; christian.stief@med.uni-muenchen.de \\ 3 Department of Radiology, University Hospital, Ludwig-Maximilians-University Munich, 80331 Munich, \\ Germany; johannes.ruebenthaler@med.uni-muenchen.de (J.R.); \\ clemens.cyran@med.uni-muenchen.de (C.C.C.) \\ * Correspondence: harun.ilhan@med.uni-muenchen.de; Tel.: +49-89-4400-77655
} Gosewisch, A.; Brosch-Lenz, J.; Gildehaus, F.J.; Zacherl, M.J.; Beyer, L.; Stief, C.G.; Holzgreve, A.; Rübenthaler, J.; et al. Correlation of an Index-Lesion-Based SPECT Dosimetry Method with Mean Tumor Dose and Clinical Outcome after ${ }^{177}$ Lu-PSMA-617 Radioligand Therapy. Diagnostics 2021, 11, 428. https://doi.org/10.3390/ diagnostics11030428

Academic Editor: Rudolf A. Werner

Received: 23 January 2021

Accepted: 25 February 2021

Published: 3 March 2021

Publisher's Note: MDPI stays neutral with regard to jurisdictional claims in published maps and institutional affiliations.

Copyright: (c) 2021 by the authors. Licensee MDPI, Basel, Switzerland. This article is an open access article distributed under the terms and conditions of the Creative Commons Attribution (CC BY) license (https:// creativecommons.org/licenses/by/ $4.0 /)$.
Abstract: Background: Dosimetry can tailor prostate-specific membrane-antigen-targeted radioligand therapy (PSMA-RLT) for metastatic castration-resistant prostate cancer (mCRPC). However, whole-body tumor dosimetry is challenging in patients with a high tumor burden. We evaluate a simplified index-lesion-based single-photon emission computed tomography (SPECT) dosimetry method in correlation with clinical outcome. Methods: 30 mCRPC patients were included (median 71 years). The dosimetry was performed for the first cycle using quantitative ${ }^{177} \mathrm{Lu}-\mathrm{SPECT}$. The response was evaluated using RECIST 1.1 and PERCIST criteria, as well as changes in PSMA-positive tumor volume (PSMA-TV) in post-therapy PSMA-PET and biochemical response according to PSA changes after two RLT cycles. Results: Mean tumor doses as well as index-lesion doses were significantly higher in PERCIST responders compared to non-responders (10.2 $\pm 12.0 \mathrm{~Gy} / \mathrm{GBq}$ vs. $4.0 \pm 2.9 \mathrm{~Gy} / \mathrm{GBq}, p=0.03$ and $13.7 \pm 14.2 \mathrm{~Gy} / \mathrm{GBq}$ vs. $5.9 \pm 4.4 \mathrm{~Gy} / \mathrm{GBq}, p=0.04$, respectively). No significant differences in mean tumor and index lesion doses were observed between responders and non-responders according to RECIST 1.1, PSMA-TV, and biochemical response criteria. Conclusion: Compared to mean tumor doses on a patient level, single index-lesion-based SPECT dosimetry correlates equally well with the response to PSMA-RLT according to PERCIST criteria and may represent a fast and feasible dosimetry approach for clinical routine.

Keywords: radioligand therapy; ${ }^{177}$ Lu-PSMA; dosimetry; SPECT; mCRPC

\section{Introduction}

Prostate-specific-membrane-antigen (PSMA) targeted theranostic approaches for metastatic castration-resistant prostate cancer (mCRPC) are of rising clinical importance [1]. PSMA-targeted radioligand therapy (PSMA-RLT) using $\beta$ - and $\alpha$-emitters, such as ${ }^{177}$ Lu- and ${ }^{225}$ Ac-labeled PSMA-617 and PSMA-I\&T, allows selective irradiation of PSMA-expressing prostate cancer (PC) cells and is used in many centers worldwide [2-8] and results of the first prospective phase 3 trial (VISION; NCT03511664) are anticipated [9].

Currently, PSMA-RLT represents a last-line therapy option in advanced $\mathrm{mCPRC}$ after exhaustion of approved therapy options, including anti-hormonal therapy, novel anti- 
hormonal agents, such as abiraterone acetate and/or enzalutamide, and taxane-based chemotherapy [10]. According to a recent meta-analysis on the efficacy and safety of ${ }^{177}$ Lu-PSMA RLT, including 17 articles and 744 patients, biochemical response with a prostate-specific antigen (PSA) decline $>50 \%$ is observed in $46 \%$ (95\% CI: $40-53 \%$ ) despite progressive disease after exhaustion of approved therapy options [11]. Nonetheless, not all patients will respond to PSMA-RLT, and biochemical progression is observed in up to $37 \%$ of patients (95\% CI: 34-40\%) despite sufficient PSMA-expression in pre-therapeutic positron emission tomography (PET) imaging [11]. Low intra-tumoral doses during ${ }^{177}$ Lu-PSMA RLT might represent one of the reasons for therapy failure despite high PSMA-expression of lesions in PSMA PET imaging. PSMA-RLT is a trade-off between sufficient tumor dose while maintaining recommended dose limits for organs at risk, including the kidneys, bone marrow, and salivary glands, which underlines the clinical importance of dosimetry during RLT [12]. However, full individual dosimetry with the segmentation of every single tumor lesion is challenging, especially in patients with a high tumor burden. Recently, Violet et al. presented data on the correlation of whole-body tumor-absorbed doses after ${ }^{177} \mathrm{Lu}$ PSMA-617 RLT with PSA response [13]. The median whole-body tumor dose was $14.1 \mathrm{~Gy}$ in patients with PSA decline $\geq$ of $50 \%$ compared to patients with a PSA decline of less than $50 \%$ with a median dose of only $9.6 \mathrm{~Gy}$, providing a rationale for individualized dosimetry during PSMA-RLT. However, tumor dosimetry, including all tumor lesions or multiple selected lesions, is elaborate and time-consuming during a clinical routine, particularly in the case of single-photon emission computed tomography (SPECT)-based dosimetry with complex tumor delineation when performed manually. This study aimed to evaluate and compare an easy and feasible, index-lesion-based SPECT dosimetry method with mean tumor doses in multiple tumor lesions on a patient level and to correlate dosimetry data with clinical outcome after ${ }^{177}$ Lu-PSMA RLT.

\section{Materials and Methods}

\subsection{Patients}

For this retrospective analysis, we included patients with mCRPC who received 2 cycles of ${ }^{177}$ Lu-PSMA-617 radioligand therapy at our institution between September 2014 and June 2018. All patients received PSMA-targeted PET combined with diagnostic computed tomography (CT) at our institution to assess PSMA-expression prior to RLT and for follow-up after two cycles of PSMA-617 RLT. Patients with different ligands for PSMA-RLT (e.g., PSMA-i\&T) were excluded to improve the comparability of dosimetry data. Patients without quantitative SPECT imaging at least three time points $(24,48,72 \mathrm{~h}$ postinjection), patients receiving only one therapy cycle were excluded from the analysis. Figure 1 provides a flowchart summarizing the criteria for patient inclusion. Indication for PSMA RLT was in accordance with the procedure guidelines for radionuclide therapy with ${ }^{177}$ Lu-labeled PSMA-ligands of the European Association of Nuclear Medicine (EANM) and with recommendations of the German Society of Nuclear Medicine [10,14]. Furthermore, indication for radioligand therapy was confirmed by the local interdisciplinary tumour board. RLT was performed in accordance with the German Medical Products Act (AMG) $\S 13.2 \mathrm{~b}$ and the updated declaration of Helsinki, § 37 (Unproven Interventions in Clinical Practice). All patients were informed about the experimental nature of this unapproved therapy as well as possible risks and side effects and provided written informed consent. The study was approved by the local ethics committee (approval number 20-178, approval date: 02.02 .2019 ). 


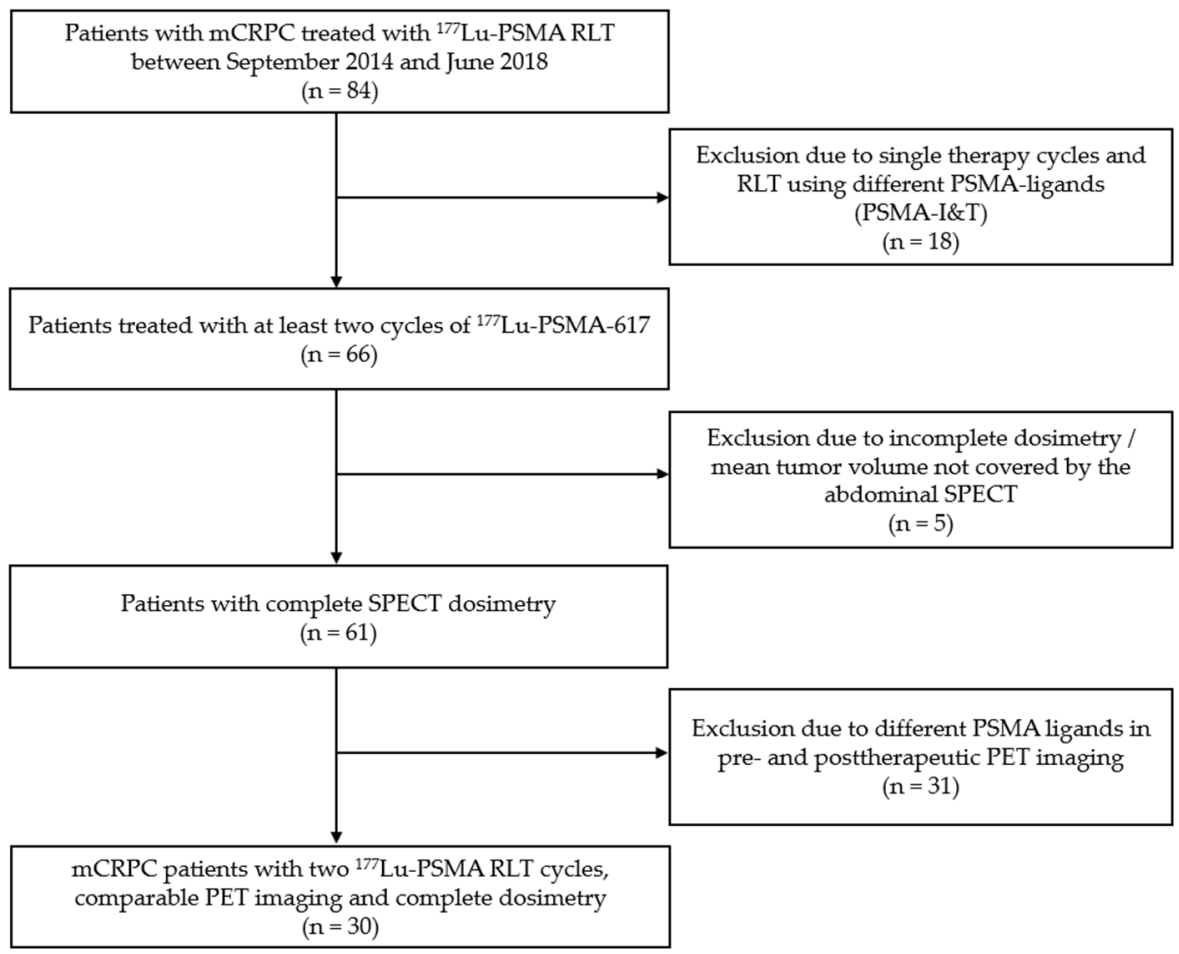

Figure 1. Flowchart with detailed information on patient selection.

\section{2. ${ }^{177}$ Lu-PSMA-617 Therapy}

${ }^{177}$ Lu-PSMA-617 was obtained by radiolabeling of DOTA-PSMA-617 precursor (ABX $\mathrm{GmbH}$, Radeberg, Germany) with no-carrier-added ${ }^{177} \mathrm{LuCl}_{3}$ (ITG GmbH, Garching, Germany) as described previously [15]. ${ }^{177} \mathrm{Lu}-\mathrm{PSMA}-617$ was injected intravenously over 15-20 min using an infusion pump. Before and after therapy, all patients were hydrated with 2 liters of sodium-iodine. During and after therapy, all patients received cool packs to reduce the blood flow of the salivary glands.

\subsection{PET/CT Imaging}

Radiolabeling and administration of ${ }^{68}$ Ga-PSMA-11 and 18F-PSMA-1007 were performed according to previously reported radiosynthesis and administration procedures [16,17]. Immediately after injection of the tracer, $20 \mathrm{mg}$ of furosemide was administered intravenously. Patients were asked to empty the bladder prior to the scan. PET/CT scans were acquired 60 min after tracer injection using an iodine-containing contrast agent (Ultravist 300, Bayer Pharma AG, Berlin, Germany; or Imeron 300, Bracco, Konstanz; Germany 2.5 mL/s) during the portal venous phase. For reconstruction, the TrueX algorithm (3 iterations, 21 subsets; Biograph 64, Siemens Healthineers, Erlangen, Germany) or the VUE Point FX algorithm (2 iterations, 36 subsets; Discovery 690) was used with an axial $168 \times 168$ matrix. Phantom studies based on the National Electrical Manufacturers Association NU2-2001 standard were conducted to allow valid pooling of results between different scanners. Mean injected activity was $213.1 \pm 49.7 \mathrm{MBq}$ in pre- and 212.6 $\pm 45.1 \mathrm{MBq}$ in post-therapeutic PET.

\subsection{Dosimetry}

Dosimetry was performed using quantitative ${ }^{177} \mathrm{Lu}-\mathrm{SPECT}$ scans of the abdomen 24,48 , and $72 \mathrm{~h}$ postinjection. The images were acquired over $15 \mathrm{~min}$ on a dual-headed Symbia T2 SPECT/CT system (Siemens Healthineers, Erlangen, Germany) as described previously $[12,15]$. For attenuation correction, a low-dose CT scan (AC-CT) was acquired together with the first SPECT scan $24 \mathrm{~h}$ postinjection. The AC-CT was coregistered on the SPECT scans acquired 48 and $72 \mathrm{~h}$ after therapy (rigid body co-registration, PMOD Version 3.609, PMOD Technologies, Zurich, Switzerland). Quantitative SPECT reconstruction em- 
ployed an in-house maximum a posteriori (MAP) algorithm with 20 iterations, 16 subsets, and a penalty factor of 0.001 , as described previously $[8,10]$. Scatter correction and resolution decompensation was applied in addition to the mentioned attenuation correction. For each patient, three to five lesions with the highest visual uptake in abdominal SPECT were evaluated. Tumor lesions were segmented semi-manually by placing volumes of interest (VOIs) with an iso-contour of $40 \%$ as proposed by Collarino et al. and confirmed by in-house phantom studies [18]. Lesion dosimetry was based on a mono-exponential fit model and mass-scaled sphere $S$-values (Figure 2).
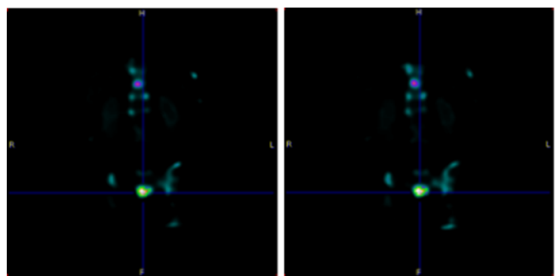

(a)

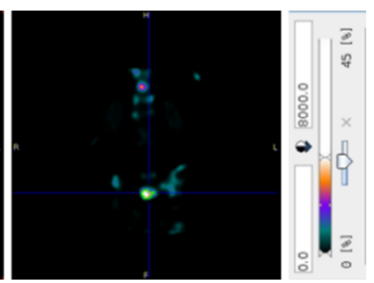

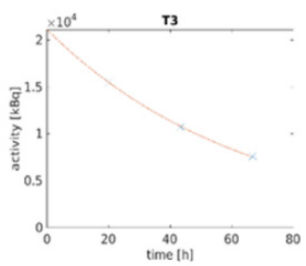

(b)

Figure 2. (a) Single-photon emission computed tomography (SPECT)/diagnostic computed tomography (CT) images at 24, 48 and $72 \mathrm{~h}$ after therapy with semi-manually placed the volume of interest (VOI) in a single bone lesion (green circle); (b) Mono-exponential fit curve of radioactivity over time of the lesion delineated in (a).

\subsection{Response Assessment}

Biochemical response assessment was based on PSA-levels according to previously described protocols [19-21]. The complete biochemical response was defined as a nonmeasurable PSA level (0 ng/mL) after PSMA RLT, partial biochemical response (PR) defined a PSA-decline $\geq 50 \%$, stable disease (SD) as a PSA change between $-50 \%$ and $+25 \%$, and progressive disease (PD) as a PSA increase $\geq 25 \%$.

Radiographic response assessment was performed according to RECIST 1.1 and modified PERCIST criteria [22,23]. RECIST 1.1 evaluation was performed on diagnostic CT images acquired during follow-up PET/CT imaging with target lesions defined as $\geq 1 \mathrm{~cm}$ for soft tissue lesions and $\geq 1.5 \mathrm{~cm}$ in shortest axis for lymph node metastases. The sum of diameter (SOD) of up to five target lesions with a maximum of two lesions per organ was evaluated. Sclerotic bone metastases were considered non-measurable lesions. PR was defined as a decrease of $\geq 30 \%$ in SOD, and PD as an increase of $\geq 20 \%$. A SOD change between a decrease of less than $30 \%$ and an increase of less than $20 \%$ was considered SD. In the case of bone-only disease, SD was considered for equivocal disease or no change. The appearance of new lesions was considered PD. For PERCIST evaluation, background activity was determined using the standardised uptake value (SUV) of a $3 \mathrm{~cm}$ diameter spherical volume of interest placed in the right side of the liver. SUVpeak of the single hottest tumor lesion was evaluated manually using a $1 \mathrm{~mL}$ VOI. The hottest tumor lesion in pre- and post-therapeutic PET did not need to be the same according to PERCIST criteria defined for FDG-PET. PR was defined as a decrease of SUVpeak of the hottest tumor lesion of $30 \%$ and a minimum of 0.8 SUV units [22]. PD was defined as an increase of SUVpeak of $30 \%$ or the occurrence of new tumor lesions. SD was defined as a decrease or increase of SUVpeak of the hottest tumor lesion between -30 and $+30 \%$ without the occurrence of new metastases.

Furthermore, changes in PSMA-positive tumor volume (PSMA-TV) after PSMA-RLT were evaluated $[21,24]$. Pre- and post-therapeutic ${ }^{68} \mathrm{Ga}-\mathrm{PSMA}-11$ PET/CT was available in 21 patients; the remaining 9 patients with 18F-PSMA-1007 PET/CT were excluded from this analysis due to different tracer biodistribution [17]. A lower threshold was defined as the mean SUV within a circular reference VOI of $2 \mathrm{~cm}$ diameter placed in the liver, avoiding the inclusion of major intrahepatic vessels based on contrast-enhanced CT images. Whole tumor volume was evaluated using a threshold-based segmentation algorithm included in Hermes Affinity v1.1 (Hermes Medical Solutions, Stockholm, Sweden), followed by 
manual correction for unspecific tracer uptake or organs with non-tumor related tracer uptakes, such as the kidneys, spleen, salivary glands, gut, and bladder. Cutoff values for therapy response in PSMA-TV assessment were defined according to previously published data [21,24]. A decline of $\geq 30 \%$ was defined as a partial response; stable disease was defined as a change of whole tumor volume between $-30 \%$ and $+30 \%$. PD in PSMA-TV assessment was defined as an increase of $\geq 30 \%$.

\subsection{Statistical Analysis}

Statistical analysis was performed using SPSS Statistics 24 (IBM, New York, NY, USA) and GraphPad Prism, Version 8.4.3 (GraphPad Software, San Diego, CA, USA). Patients were divided into subgroups based on treatment response. Estimated lesion absorbed doses were compared to therapy outcome, which was determined based on image response assessment using PERCIST criteria, RECIST 1.1 criteria, and PSMA-TV applied on preand post-therapeutic ${ }^{68} \mathrm{Ga}$-PSMA PET/CT as well as biochemical PSA-response. Patients were grouped as non-responders in the case of SD and PD and as responders in the case of CR or PR. Gaussian distribution was evaluated using the Shapiro-Wilk test. For analysis of variance between subgroups, Mann-Whitney $U$ test was used. Receiver operating characteristic analysis (ROC analysis) was performed for mean dose and the dose of indexlesions in therapy response evaluations. The cutoff doses showing the maximum sum of sensitivity and specificity in ROC analysis were selected as threshold doses. The frequency of responders and non-responders above and below the determined threshold value were compared using the chi-squared test.

\section{Results}

\subsection{Patient Characteristics}

Thirty patients with mCRPC (mean age of $71.4 \pm 9.9$ ) were included. Detailed patient characteristics are provided in Table 1. Median injected activity for the first and second therapy cycle were $6.01 \mathrm{GBq} \pm 0.8$ and $6.00 \mathrm{GBq} \pm 0.9$, respectively.

Table 1. Initial clinical characteristics of the included patients.

\begin{tabular}{ccc}
\hline Initial Clinical Characteristics & \multicolumn{2}{c}{ Total Patients $=\mathbf{3 0}$} \\
\hline Age (years) & 71.4 & $(52.4-91.2)$ \\
Median (range) & & $(77 \%)$ \\
Previous therapies ( $\boldsymbol{n})$ & 23 & $(63 \%)$ \\
Radical prostatectomy & 19 & $(90 \%)$ \\
Radiotherapy & 27 & $(3 \%)$ \\
Androgen deprivation therapy & 1 & $(63 \%)$ \\
Brachytherapy & 19 & $(30 \%)$ \\
Abiraterone/enzalutamide & 9 & $(50 \%)$ \\
Radium-223 & 15 & $(1.4-9237.0)$ \\
Chemotherapy (docetaxel and/or cabazitaxel) & & $(6-10)$ \\
\hline Serum PSA baseline (ng/mL) & 129.0 & $(90 \%)$ \\
Median (range) & & $(77 \%)$ \\
Gleason score $(\boldsymbol{n}=\mathbf{2 3})$ & 9 & $(10 \%)$ \\
Median (range) & & $(7 \%)$ \\
Metastases localization $(\boldsymbol{n})$ & 27 & $(17 \%)$ \\
Bone & 23 & $(0.6-16.7)$ \\
\hline Lymph nodes & 3 & \\
Liver & 2 & 5 \\
Lungs & 5.4 & \\
Local recurrence & &
\end{tabular}


Table 1. Cont.

\begin{tabular}{ccc}
\hline Initial Clinical Characteristics & \multicolumn{2}{c}{ Total Patients $=30$} \\
\hline $\begin{array}{c}\text { Interval 1st-2nd therapy cycle } \\
\text { Median (range) (weeks) }\end{array}$ & 8 & $(4.3-10.1)$ \\
\hline $\begin{array}{c}\text { Interval 1st therapy cycle-follow-up PET } \\
\text { Median (range) (weeks) }\end{array}$ & 17.1 & $(13.1-22.2)$ \\
\hline
\end{tabular}

\subsection{Biochemical Response Assessment}

Median PSA decline after two PSMA-RLT cycles was -37.4\% (range: -99.6 to $730.3 \%$ compared to baseline PSA). 13/30 (43\%) patients were categorized as responders and showed a PSA decline $\geq$ of $50 \%$ (PR), the remaining 17 patients $(57 \%)$ were grouped as non-responders, with 9 patients (30\%) showing a PSA change between $\leq-50 \%$ and $+25 \%$ (SD) and 8 patients (27\%) showing a PSA increase $\geq 25 \%$ (PD).

\subsection{Therapy Response Assessment Using PERCIST and RECIST 1.1 Criteria}

According to RECIST 1.1, PR was observed in 2/30 (7\%), SD in 8/30 (26\%), and PD in 20/30 patients (67\%). According to PERCIST, PR was observed in 10/30 (33\%) and PD in $20 / 30$ patients $(67 \%)$. No patient showed SD according to PERCIST criteria. Response assessment evaluating PSMA-TV showed PR in 7/21 (33\%), SD in 6/21 (29\%), and PD in $8 / 21$ patients $(38 \%)$.

Comparing the results of PERCIST and RECIST evaluations, for 21 of 30 patients (70\%), PERCIST and RECIST matched. In $7 / 9$ patients with a mismatch between PERCIST and RECIST, a significant reduction of PSMA expression in skeletal target lesions resulted in PR according to PERCIST criteria, while bone metastases were non-measurable according to RECIST without unequivocal progression resulting in SD. One patient showed an increasing SUVpeak of bone metastases resulting in PD according to PERCIST, while the RECIST evaluation showed SD. Another patient with mismatching results in PERCIST and RECIST evaluation showed a significantly growing cardiophrenic lymph node metastasis, while SUVpeak of this metastasis was stable and other lymph node metastases were also significantly decreasing in size and PSMA-expression, resulting in PR according to PERCIST and PD according to RECIST 1.1 (Figure 3).

Comparing PSMA-TV with PERCIST and RECIST criteria, response assessment using PSMA-TV showed matching results with PERCIST and RECIST 1.1 evaluation in 14/21 $(67 \%)$ and $11 / 21(52 \%)$ patients, respectively. A detailed comparison of radiographic response according to different criteria is provided in Table 2.

Table 2. Radiographic response assessment evaluating prostate-specific membrane-antigen-targeted radioligand therapy (PSMA-TV) in 21 patients compared to PERCIST and RECIST 1.1 evaluation. PD: progressive disease; SD: stable disease; PR: partial response.

\begin{tabular}{ccccc}
\hline \multirow{2}{*}{} & & \multicolumn{3}{c}{ PSMA-TV } \\
\cline { 3 - 5 } & & PD & SD & PR \\
\hline \multirow{2}{*}{ PERCIST } & PD & $8(38 \%)$ & $3(14 \%)$ & $1(5 \%)$ \\
& SD & $0(0 \%)$ & $0(0 \%)$ & $0(0 \%)$ \\
& PR & $0(0 \%)$ & $3(14 \%)$ & $6(29 \%)$ \\
\hline \multirow{2}{*}{ RECIST 1.1 } & PD & $7(33 \%)$ & $4(19 \%)$ & $1(5 \%)$ \\
& SD & $1(5 \%)$ & $2(10 \%)$ & $4(19 \%)$ \\
& PR & $0(0 \%)$ & $0(0 \%)$ & $2(10 \%)$ \\
\hline
\end{tabular}




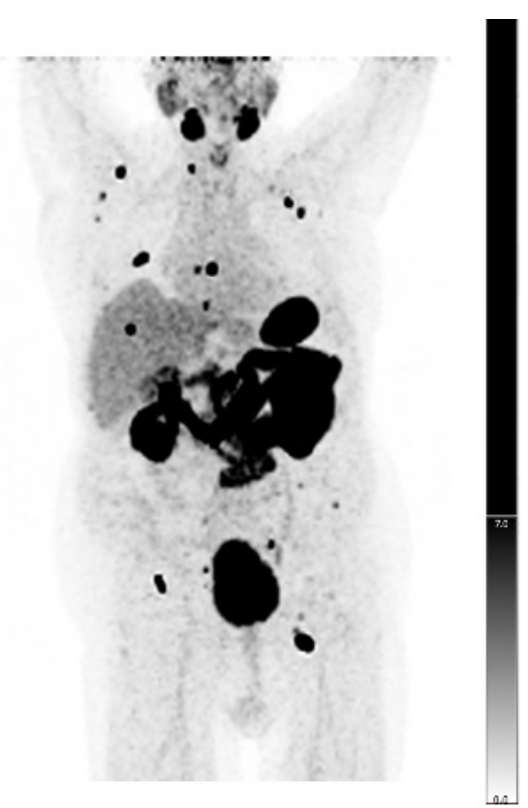

(a) Pre-therapeutic ${ }^{68} \mathrm{Ga}$-PSMA-11 PET

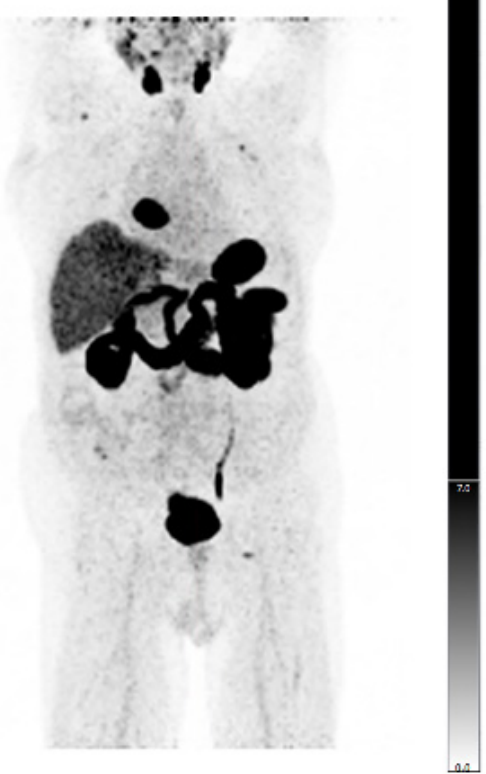

(b) post-therapeutic ${ }^{68} \mathrm{Ga}-\mathrm{PSMA}-11$ PET

Figure 3. Pre- (a) and post-therapeutic PSMA-11 PET/CT (b) of an mCRPC patient with metastatic disease in bones and lymph nodes. Follow-up was performed after two cycles of ${ }^{177} \mathrm{Lu}-\mathrm{PSMA}-617$ RLT. Partial response was observed according to PERCIST criteria, and progressive disease according to RECIST 1.1 due to the significantly increased size of a cardiophrenic lymph node metastasis, which was selected as a measurable target lesion in pre-therapeutic CT. SPECT dosimetry showed a mean dose of $5.4 \mathrm{~Gy} / \mathrm{GBq}$ and a dose of $6.2 \mathrm{~Gy} / \mathrm{GBq}$ for the index-lesion with the highest uptake.

\subsection{Comparison of Image-Based and-Biochemical Response Evaluation}

Results of radiographic response assessment and biochemical response evaluation are listed in detail in Table 3. According to PERCIST criteria, there was a good correlation with biochemical response assessment in $17 / 30$ patients $(57 \%)$, with 8 patients showing PD and 9 PR. PD, according to PERCIST, despite PR in biochemical response assessment, was observed in three patients due to the occurrence of new metastases and rising SUVpeak in one patient. Mean PSA decline in patients with PR according to PERCIST criteria was $81.0 \pm 21.3 \%$ compared to a mean increase of $85.2 \pm 229.5 \%$ in patients with PD $(p<0.001)$ RECIST evaluation was in concordance with biochemical response assessment in 11/30 $(37 \%)$ cases, including 8 patients with PD, one with SD, and two with PR. PSMA-TV response assessment revealed concordant biochemical response in 12/21 patients (57\%). While patients with PR showed a PSA decline of $73.4 \pm 24.6 \%$, patients with PD showed a PSA increase of $147.7 \pm 286.4 \%(p<0.01)$.

Table 3. Results of biochemical response assessment and image response assessment evaluated using PERCIST criteria in PET and RECIST criteria in CT in 30 patients.

\begin{tabular}{ccccccccccc}
\hline & & \multicolumn{3}{c}{ PERCIST } & \multicolumn{3}{c}{ RECIST } & \multicolumn{3}{c}{ PSMA-TV } \\
\cline { 3 - 10 } & & PD & SD & PR & PD & SD & PR & PD & SD & PR \\
\hline \multirow{3}{*}{ PSA } & PD & $8(27 \%)$ & $0(0 \%)$ & $0(0 \%)$ & $8(27 \%)$ & $0(0 \%)$ & $0(0 \%)$ & $4(19 \%)$ & $1(5 \%)$ & $0(0 \%)$ \\
& SD & $8(27 \%)$ & $0(0 \%)$ & $1(3 \%)$ & $8(27 \%)$ & $1(3 \%)$ & $0(0 \%)$ & $3(14 \%)$ & $2(10 \%)$ & $1(10 \%)$ \\
& PR & $4(13 \%)$ & $0(0 \%)$ & $9(27 \%)$ & $4(13 \%)$ & $7(27 \%)$ & $2(7 \%)$ & $1(5 \%)$ & $3(14 \%)$ & $6(29 \%)$ \\
\hline
\end{tabular}




\subsection{Dosimetry Results}

Dosimetry was performed for multiple tumor lesions as a correlate for mean tumor dose on a patient-level in a total of 141 tumor lesions ( 97 bone metastases, 44 lymph node metastases, median 5 lesions per patient). The mean tumor dose was $5.7 \pm 6.4 \mathrm{~Gy} / \mathrm{GBq}$ with a mean absorbed dose of $7.7 \pm 9.7 \mathrm{~Gy} / \mathrm{GBq}$ and $4.7 \pm 3.9 \mathrm{~Gy} / \mathrm{GBq}$ in lymph node and bone metastases, respectively. The mean index-lesion-based dosimetry was calculated for single tumor lesions on a patient level. The mean dose in the index-lesion-based method was $8.5 \pm 9.4 \mathrm{~Gy} / \mathrm{GBq}$.

\subsubsection{Dose Estimations of Biochemical Responders and Non-Responders}

There was no significant difference regarding the lesion absorbed doses in biochemical responders (13/30 patients, $43 \%)$ and non-responders (17/30 patients, $57 \%)$ with a mean tumor dose of $8.3 \pm 10.1 \mathrm{~Gy} / \mathrm{GBq}$ vs. $4.3 \pm 3.3 \mathrm{~Gy} / \mathrm{GBq}(p=0.21$; Figure $4 \mathrm{a})$. Similarly, no significant differences were observed for index-lesion dosimetry (biochemical responders: $11.7 \pm 12.9 \mathrm{~Gy} / \mathrm{GBq}$ vs. biochemical non-responders: $6.1 \pm 4.7 \mathrm{~Gy} / \mathrm{GBq} ; p=0.12$; Figure $4 \mathrm{~b}$ ).

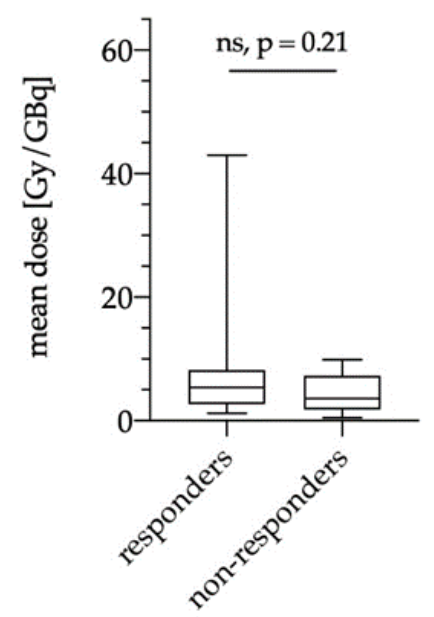

(a)

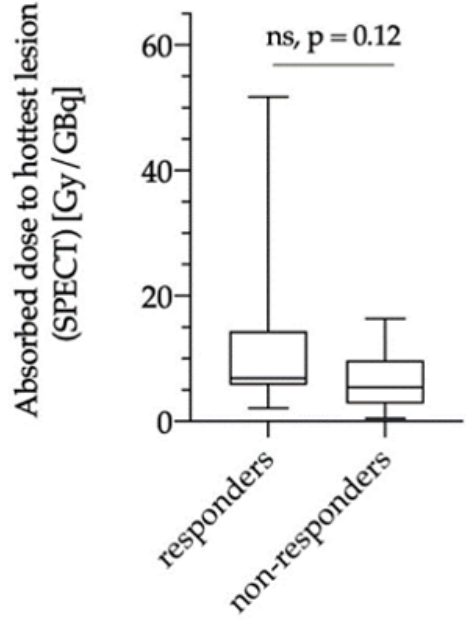

(b)

Figure 4. Comparison of mean absorbed doses in biochemical responders and non-responders using SPECT dosimetry for averaged tumor lesions on a patient-level ("mean dose", (a)) and for a single lesion with highest uptake ("hottest lesion", (b)) in the index-lesion-based dosimetry method. ns $=$ not significant.

3.5.2. Dose Estimations for Responders and Non-responders as Evaluated via PERCIST, RECIST and PSMA-TV

The averaged mean tumor dose was significantly higher in responders according to PERCIST criteria (10/30; patients 33\%) compared to non-responders (20/30 patients; $67 \%)$ with $10.2 \pm 12.0 \mathrm{~Gy} / \mathrm{GBq}$ vs. $4.0 \pm 2.9 \mathrm{~Gy} / \mathrm{GBq}, p=0.03$ (Figure $5 \mathrm{a}$ ). According to RECIST 1.1 , no significant differences were observed between responders and non-responders $(25.0 \pm 25.5 \mathrm{~Gy} / \mathrm{GBq}$ vs. $4.7 \pm 3.3 \mathrm{~Gy} / \mathrm{GBq}, p=0.11)$; however, only $2 / 30$ patients were grouped as responders, whereas $28 / 30$ patients showed SD and PD (Figure $5 b$ ). Responders according to PSMA-TV evaluation (7/21 patients $33 \%$ ) had a mean averaged tumor dose of $10.7 \pm 14.5 \mathrm{~Gy} / \mathrm{GBq}$ compared to non-responders (14/21 patients $67 \%)$ with a mean tumor dose of $4.5 \pm 3.6 \mathrm{~Gy} / \mathrm{GBq}(p=0.32)$ (Figure $5 c$ ). 


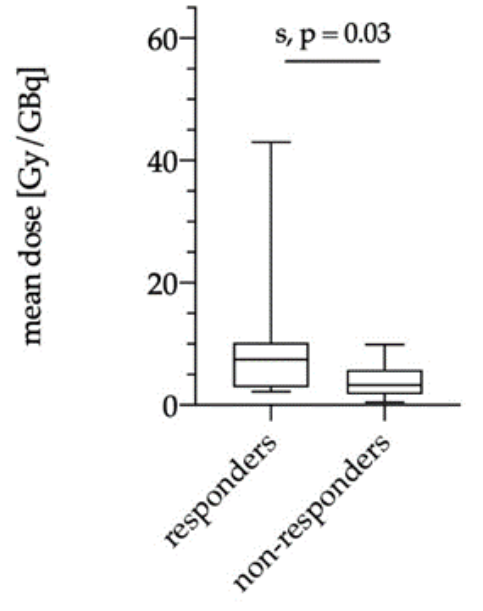

(a) PERCIST

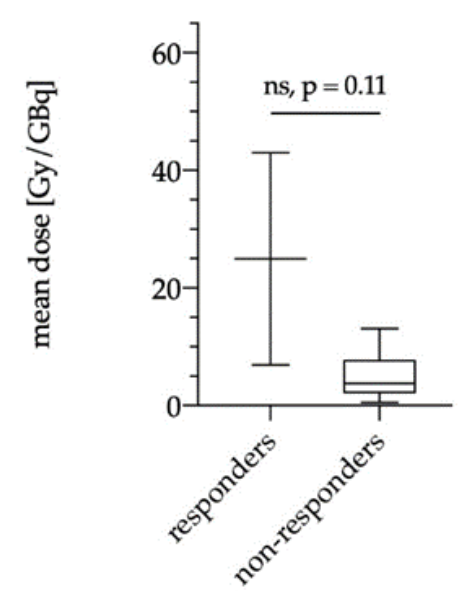

(b) RECIST

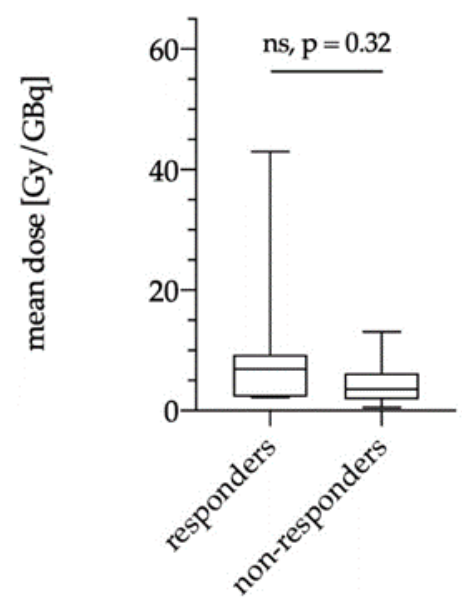

(c) PSMA-TV

Figure 5. Mean absorbed tumor dose for averaged multiple lesions ("mean dose" in Gy/GBq) stratified according to responders and non-responders using modified PERCIST criteria (a), RECIST 1.1 criteria (b), and PSMA-TV ( $n=21)$ (c) evaluated. $\mathrm{s}=$ significant, $\mathrm{ns}=$ not significant.

Similar results were observed for the index-lesion-based analysis with significantly higher tumor doses in hottest lesions of responders according to PERCIST criteria (mean: $13.7 \pm 14.2 \mathrm{~Gy} / \mathrm{GBq}$ ) compared to non-responders (mean: $5.9 \pm 4.4 \mathrm{~Gy} / \mathrm{GBq} ; p=0.04$; Figure 6a). Responders and non-responders according to RECIST 1.1 criteria and PSMA-TV assessment did not show significant differences with mean index-lesion absorbed doses of $30.1 \pm 30.6 \mathrm{~Gy} / \mathrm{GBq}$ vs. $7.0 \pm 4.9 \mathrm{~Gy} / \mathrm{GBq}(p=0.11)$ and $13.8 \pm 17.0 \mathrm{~Gy} / \mathrm{GBq}$ vs. $6.6 \pm 5.3 \mathrm{~Gy} / \mathrm{GBq}(p=0.29)$, respectively (Figure $6 \mathrm{~b}, \mathrm{c})$.

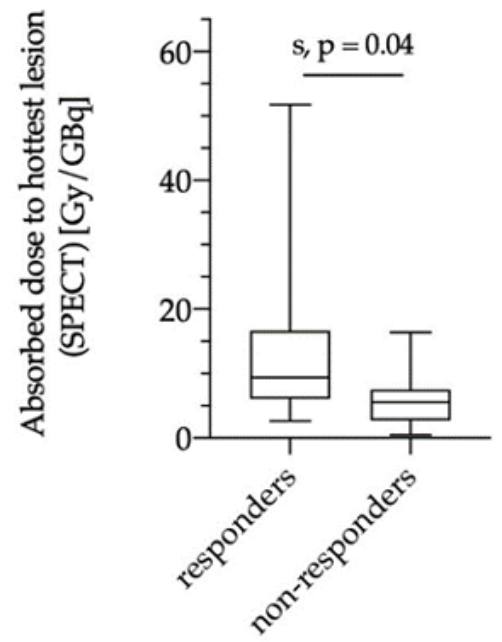

(a) PERCIST

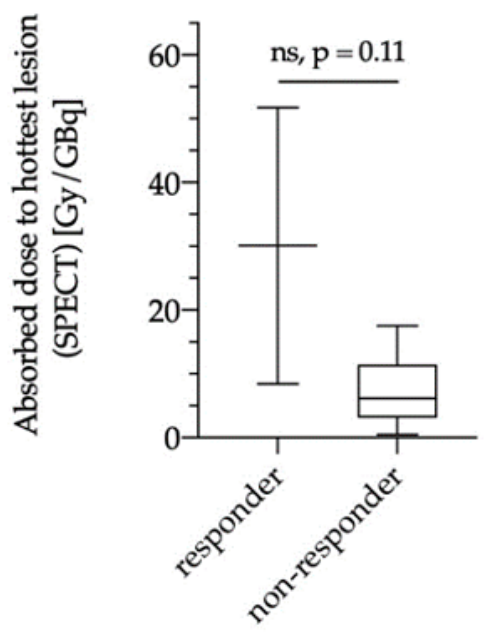

(b) RECIST

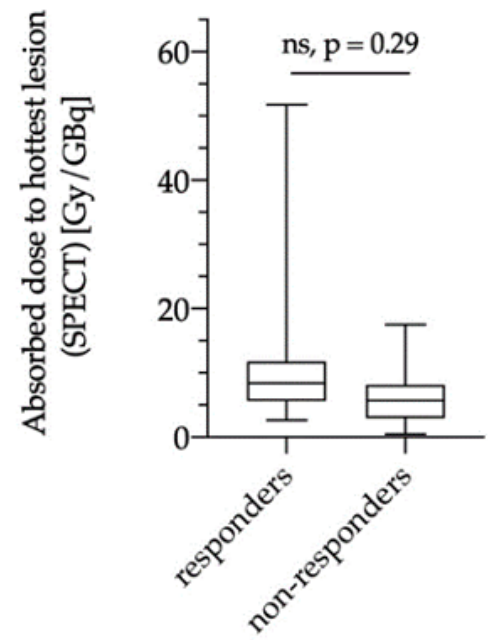

(c) PSMA-TV

Figure 6. Index-lesion-based dosimetry for single tumor lesions with the highest uptake ("hottest lesion" in Gy/GBq) stratified according to responders and non-responders using modified PERCIST criteria (a), RECIST 1.1 criteria (b), and PSMA-TV $(n=21)(\mathbf{c}) . \mathrm{s}=$ significant, $\mathrm{ns}=$ not significant.

ROC-analysis showed a significant C-index for mean tumor volume dose and indexlesion dose in responders and non-responders according to PERCIST criteria (0.75 and 0.86; $p=0.03$ and $p=0.04$, respectively; Figure 7). Threshold values of mean dose and the dose of the index-lesions were 5.0 Gy/GBq and 5.8 Gy/GBq according to PERCIST and 6.5 and 8.0 Gy/GBq according to RECIST. The frequency of therapy response was significantly 
higher in patients with mean and index-lesion tumor doses above the determined threshold values compared to patients with lower doses (PERCIST: 16.7\% vs. 58.3\% for mean tumor volume dose and $8.3 \%$ vs. $50 \%$ for index tumor lesion dose) and RECIST analysis ( $0 \%$ vs. $20 \%$ for mean tumor volume dose and index tumor lesion dose, respectively).

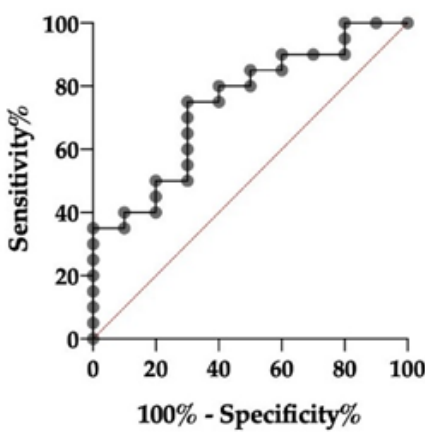

(a) mean dose

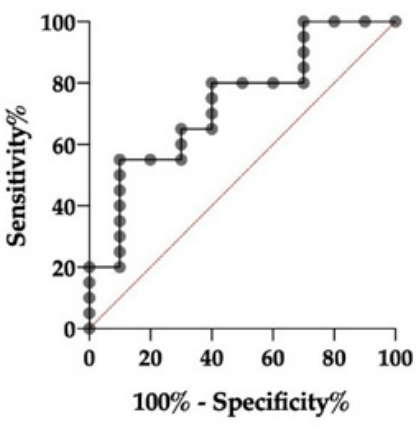

(b) dose of the hottest lesions

Figure 7. Receiver operating characteristics curve of averaged tumor lesions ("mean dose", (a)) and the index-lesion-based analysis ("hottest lesion", (b)) for responders and non-responders according to PERCIST criteria mean with a C-index of 0.75 and a $p$-value of 0.03 for the mean dose and a-with-a C-index of 0.86 and a $p$-value of 0.04 for the hottest-lesion dosimetry, respectively.

\section{Discussion}

Novel therapeutic agents from chemotherapy to second-line anti-hormonal agents and radioligand therapy using ${ }^{177} \mathrm{Lu}$ - or ${ }^{225} \mathrm{Ac}$-labeled PSMA ligands changed the landscape of available therapy options for $\mathrm{mCRPC}$ [25]. Results of the first phase 3 trial comparing ${ }^{177} \mathrm{Lu}$ PSMA-617 with best supportive care in MCRPC patients after taxane-based chemotherapy (VISION; NCT03511664) might expand the armamentarium for mCRPC with a novel approved radiopharmaceutical besides ${ }^{223}$ Radium-dichloride $\left({ }^{223} \mathrm{Ra}\right)[9,26]$. However, unlike ${ }^{223} \mathrm{Ra}$, which targets bone metastases only, ${ }^{177} \mathrm{Lu}-\mathrm{PSMA}$-ligands represent a systemic therapy option targeting all PSMA-avid tumor lesions. Theranostic concepts for prostate cancer include pre-therapeutic visualization and verification of PSMA-expression prior to RLT by PSMA-PET, as well as pre- and peri-therapeutic dosimetry [1]. However, the clinical implementation of individualized dosimetry for prostate cancer theranostics is still a matter of debate, and it remains unclear whether dosimetry data will improve patient management, especially when considering additional requirements and efforts, including standardization and harmonization of therapy and dosimetry protocols [27,28]. Furthermore, it is mandatory to evaluate dosimetry results not only in relation to RLT associated toxicity but also with therapy outcome.

In this study, we evaluate dosimetry for the first RLT cycle using ${ }^{177}$ Lu-PSMA-617 in correlation with outcome parameters, including radiographic response in PSMA-PET and diagnostic CT according to RECIST 1.1, PERCIST, and changes in PSMA-TV as well as biochemical response according to changes in PSA values. We observed PSA decline $\geq 50 \%$ in $43 \%$ of patients after 2 PSMA-RLT cycles with a median cumulative activity of $12 \mathrm{GBq}$. These results are comparable to other groups as demonstrated in a metaanalysis summarizing 10 studies published between 2015 and 2018, including a total of 455 patients with $34.5 \%$ showing a PSA decline $\geq$ of $50 \%$ after PSMA-RLT. Several groups demonstrated the feasibility of response assessment based on PSMA-PET-based parameters in correlation with PSA-response, including modified PERCIST as well as PSMA-TV after RLT and/or chemotherapy [21,24], whereas response assessment in mCRPC using RECIST 1.1 criteria is hampered by non-measurable disease in case of frequently observed bone metastases [29,30]. We observed similar results in our cohort with higher concordance rates between biochemical response and PSMA-PET-based criteria as opposed to morphological RECIST 1.1 criteria (Table 3). Despite PERCIST being validated for FDG-PET only, a concordance rate of 57\% indicates that PERCIST criteria might also be 
applicable for response assessment in prostate cancer. Nonetheless, larger cohorts and preferably prospective trials are necessary to confirm this hypothesis. Similar to PERCIST, a concordance rate of $57 \%$ was also observed between biochemical response and response according to PSMA-TV, whereas the correlation rate between biochemical response and response according to RECIST 1.1 criteria was only $37 \%$. The relevance of molecular PET parameters in prostate cancer was also highlighted in a recently published consensus statement on PSMA PET/CT-based response assessment, emphasizing the value of semiquantitative PET parameters, such as standardized uptake value (SUV) and quantification of PSMA-avid tumor burden [31]. Nonetheless, the discordance of biochemical response and radiographic response according to PERCIST and PSMA-TV was observed in $43 \%$ of our cohort, respectively. PERCIST showed PD in 12 patients despite biochemical SD in 8 and PR 4 patients. One of the main reasons for this discrepancy is the fact that all new lesions are considered as PD according to PERCIST criteria [22]. However, this approach might not be appropriate in mCRPC and RLT, where the response is observed in PSMA-avid lesions, and new lesions, particularly with small volume, might not impact patient outcome [32]. These patients can be considered as mixed responders [32]. However, the mixed response was not included in the present analysis considering our relatively small cohort of 30 patients. Nonetheless, these considerations must be taken into account when evaluating dosimetry data in correlation with response to RLT. The ideal metric for response assessment after RLT is yet to be defined, particularly as serum PSA can be limited in patients receiving androgen-deprivation therapy. Furthermore, for radionuclide therapy, using ${ }^{223} \operatorname{Ra}$ PSA is of limited prognostic value in contrast to changes in alkaline phosphatase and lactate dehydrogenase levels [33]. Thus, radiographic response assessment implementing PSMAbased semi-quantitative parameters will gain increasing relevance in mCRPC. This includes therapy-associated changes in PSMA-avid tumor volume, which has been demonstrated for ${ }^{68} \mathrm{Ga}$-labeled PSMA ligands [21]. Nine patients with 18F-PSMA-1007 PET have been excluded from PSMA-TV analysis, as different biodistribution will affect tumor delineation using cutoff values established for ${ }^{68} \mathrm{Ga}$-PSMA ligands. Further trials investigating different PSMA-ligands labeled with ${ }^{68} \mathrm{Ga}$ and $18 \mathrm{~F}$, even, including phantom measurements for pooling of different tracers and scanners, are needed to further establish the value of PSMA-PET during follow-up after RLT.

Recently, Violet et al. presented encouraging data on the value of SPECT dosimetry in 30 patients receiving up to four cycles of ${ }^{177} \mathrm{Lu}$-PSMA-617 within a prospective trial [13]. SPECT-based tumor dosimetry was performed for the whole tumor burden and correlated with PSA response at 12 weeks with a significantly higher mean dose for "whole-body" tumor lesions in patients with PSA decline $\geq 50 \%$ compared to patients with a decline $<50 \%$ (14.1 Gy vs. $9.6 \mathrm{~Gy} ; p<0.01$ ). This was not observed when considering indexlesion tumor dose (numbers not shown; $p=0.09$ ). In the present analysis, dosimetry was performed with two different approaches on a patient level to evaluate a clinically feasible and applicable, time-saving method for peri-therapeutic RLT dosimetry during daily routine. The dosimetry was performed for multiple lesions per patient to assess the mean tumor dose as well as for a single index-lesion with the highest uptake on ${ }^{177} \mathrm{Lu}$ PSMA-617 SPECT. In contrast to Violet et al., we did not observe significant differences between dosimetry data of biochemical responders and non-responders (Figure 4) with a mean tumor dose of $8.3 \mathrm{~Gy} / \mathrm{GBq}$ for responders and $4.3 \mathrm{~Gy} / \mathrm{GBq}$ for non-responders $(p=0.21)$. Similar results were observed for index-lesion-based dosimetry (11.7 Gy/GBq vs. $6.1 \mathrm{~Gy} / \mathrm{GBq} ; p=0.12$ ). These contradictory results might be explained by differences in the patient population and therapy protocols. Violet et al. excluded patients with single FDG-avid tumor lesions with concomitant low PSMA-expression. FDG-PET in mCRPC patients is not routinely performed prior to RLT at our site. Therefore, we cannot rule out whether patients with FDG-avid tumor lesions were included or not. However, in agreement with other groups, we believe that PSMA-RLT might still be successful in patients with PSMA-avid disease despite single FDG-avid lesions [34]. Furthermore, Violet et al. performed up to four RLT cycles with a mean activity of $7.8 \mathrm{GBq}$, whereas 
we performed two cycles with a mean activity of $6 \mathrm{GBq}$ per cycle. It remains unclear whether higher therapy doses and cumulative activities would have resulted in higher differences regarding dosimetry data of biochemical responders and non-responders in our cohort. Nonetheless, Rathke et al. demonstrated a tendency for a positive dose-response relationship in mCRPC patients treated with activities between $4,6,7.4$, and $9.3 \mathrm{GBq}{ }^{177} \mathrm{Lu}$ PSMA-617 with higher rates of partial remission in patients receiving higher treatment activities, whereas initial PSA response showed no correlation with treatment activity [35]. This also indicates that initial PSA response at a predefined time point might not be the ideal metric for response assessment after RLT, which is also confirmed by the fact that delayed PSA-response can be observed in up to $29 \%$ when performing a second or third RLT cycle [3]. Nonetheless, it remains important to identify non-responders at early-stages in order not to delay alternative therapy options. When considering response according to PERCIST criteria, we could demonstrate that dosimetry for the first therapy cycle correlates with response to RLT (Figures 5 and 6). Both mean tumor dose, as well as index-lesionbased tumor dose, was significantly higher in responders compared to non-responders. Furthermore, ROC-analysis provided cutoff values for successful therapy. Despite having analyzed differences in tumor dose between responders and non-responders according to RECIST 1.1 criteria, the interpretation of these results is highly limited due to the very low number of responders (2/30 patients, $7 \%$ ), which once again emphasizes that RECIST 1.1 does not represent the best response criteria after PSMA-RLT.

Nonetheless, considering our results for PERCIST responders and non-responders, index-lesion-based dosimetry might represent a feasible and simple dosimetry approach for clinical routine and has the potential to impact patient management as early as at the time of the first therapy cycle. However, PSA-changes and clinical factors, including quality of life, still have to be taken into account when considering changes in management. Furthermore, dosimetry data for single-lesions has to be interpreted with caution, especially when considering the impact of heterogeneity and variance of single lesions on dosimetry with an uncertainty ranging from $14 \%$ to $102 \%$ for single index-lesions [36], which also represents one of the main limitations of our analysis. Further limitations include the retrospective design of this study and the relatively small sample size despite having performed PSMA-RLT in a considerably larger cohort. However, patients without SPECT data at least three time points after RLT, as well as patients where RLT was performed using a different PSMA ligand (e.g., PSMA-I\&T), were excluded for improved comparison and interpretation of our data.

\section{Conclusions}

Peri-therapeutic dosimetry during the first cycle of PSMA-RLT correlates with therapy response according to PERCIST criteria. Both mean tumor dose for multiple lesions on a patient level and single index-lesion-based SPECT dosimetry show significantly higher dose values in responders compared to non-responders indicating that the index-lesionbased dosimetry method may represent a fast and feasible dosimetry method during the clinical routine.

Author Contributions: Conceived and designed the method: F.V., A.G., J.B.-L., F.J.G., G.B., A.T., H.I. Evaluated and analyzed the data: F.V., L.M., A.G., J.B.-L., G.B., H.I. Participated in data interpretation: M.J.Z., L.B., A.H., J.R., C.C.C., P.B. Wrote the manuscript: F.V., H.I. Critically revised the manuscript: C.G.S., J.R., C.C.C., P.B., A.T. All authors have read and agreed to the published version of the manuscript.

Funding: J.B.-L. was partly funded by the Deutsche Forschungsgemeinschaft (DFG); Research Training Group GRK 2274, project number 299102935); H.I. was supported by grants from the Ludwig-Maximilians-University (FöFoLe program).

Institutional Review Board Statement: The retrospective study was conducted according to the guidelines of the Declaration of Helsinki, and approved by the local Ethics Committee (Medical Faculty of the LMU Munich; approval number 20-178; approval date 02.02.2019). 
Informed Consent Statement: Patient consent was waived due to the retrospective design of this study. The requirement to obtain informed consent can be waived for irreversibly anonymized patient data per our local Ethics committee (Medical Faculty of the LMU Munich).

Data Availability Statement: The data presented in this study can be made available from the corresponding author upon reasonable request.

Conflicts of Interest: The authors declare no conflict of interest.

\section{References}

1. O'Dwyer, E.; Bodei, L.; Morris, M.J. The role of theranostics in prostate cancer. Semin. Radiat. Oncol. 2021, 31, 71-82. [CrossRef]

2. Ahmadzadehfar, H.; Rahbar, K.; Kürpig, S.; Bögemann, M.; Claesener, M.; Eppard, E.; Gärtner, F.; Rogenhofer, S.; Schäfers, M.; Essler, M. Early side effects and first results of radioligand therapy with $\left({ }^{177}\right)$ Lu-DKFZ-617 PSMA of castrate-resistant metastatic prostate cancer: A two-centre study. EJNMMI Res. 2015, 5, 114. [CrossRef]

3. Rahbar, K.B.M.; Yordanova, A.; Eveslage, M.; Schäfers, M.; Essler, M.; Ahmadzadehfar, H. Delayed response after repeated ${ }^{177}$ Lu-PSMA-617 radioligand therapy in patients with metastatic castration resistant prostate cancer. Eur. J. Nucl. Med. Mol. Imaging 2018, 45, 243-246. [CrossRef]

4. Yadav, M.P.; Ballal, S.; Tripathi, M.; Damle, N.A.; Sahoo, R.K.; Seth, A.; Bal, C. ${ }^{177}$ Lu-DKFZ-PSMA-617 therapy in metastatic castration resistant prostate cancer: Safety, efficacy, and quality of life assessment. Eur. J. Nucl. Med. Mol. Imaging 2017, 44, 81-91. [CrossRef]

5. Barber, T.W.; Singh, A.; Kulkarni, H.R.; Niepsch, K.; Billah, B.; Baum, R.P. Clinical outcomes of ${ }^{177}$ Lu-PSMA radioligand therapy in earlier and later phases of metastatic castration-resistant prostate cancer grouped by previous taxane chemotherapy. J. Nucl. Med. 2019, 60, 955-962. [CrossRef] [PubMed]

6. Baum, R.P.; Kulkarni, H.R.; Schuchardt, C.; Singh, A.; Wirtz, M.; Wiessalla, S.; Schottelius, M.; Mueller, D.; Klette, I.; Wester, H. ${ }^{177} \mathrm{Lu}$-labeled prostate-specific membrane antigen radioligand therapy of metastatic castration-resistant prostate cancer: Safety and efficacy. J. Nucl. Med. 2016, 57, 1006-1013. [CrossRef] [PubMed]

7. Von Eyben, F.E.; Roviello, G.; Kiljunen, T.; Uprimny, C.; Virgolini, I.; Kairemo, K.; Joensuu, T. Third-line treatment and ${ }^{177}$ LuPSMA radioligand therapy of metastatic castration-resistant prostate cancer: A systematic review. Eur. J. Nucl. Med. Mol. Imaging 2018, 45, 496-508. [CrossRef]

8. Hofman, M.S.; Violet, J.; Hicks, R.J.; Ferdinandus, J.; Thang, S.P.; Akhurst, T.; Iravani, A.; Kong, G.; Kumar, A.R.; Murphy, D.G.; et al. [177 Lu]-PSMA-617 radionuclide treatment in patients with metastatic castration-resistant prostate cancer (LuPSMA trial): A single-centre, single-arm, phase 2 study. Lancet Oncol. 2018, 19, 825-833. [CrossRef]

9. Rahbar, K.; Bodei, L.; Morris, M.J. Is the vision of radioligand therapy for prostate cancer becoming a reality? An overview of the phase III VISION trial and its importance for the future of theranostics. J. Nucl. Med. 2019, 60, 1504-1506. [CrossRef] [PubMed]

10. Kratochwil, C.; Fendler, W.P.; Eiber, M.; Baum, R.; Bozkurt, M.F.; Czernin, J.; Delgado Bolton, R.C.; Ezziddin, S.; Forrer, F.; Hicks, R.J.; et al. EANM procedure guidelines for radionuclide therapy with ${ }^{177} \mathrm{Lu}$-labelled PSMA-ligands ( ${ }^{177}$ Lu-PSMA-RLT). Eur. J. Nucl. Med. Mol. Imaging 2019, 46, 2536-2544. [CrossRef] [PubMed]

11. Yadav, M.P.; Ballal, S.; Sahoo, R.K.; Dwivedi, S.N.; Bal, C. Radioligand therapy with ${ }^{177}$ Lu-PSMA for metastatic castration-resistant prostate cancer: A systematic review and meta-analysis. AJR Am. J. Roentgenol. 2019, 213, 275-285. [CrossRef] [PubMed]

12. Delker, A.; Fendler, W.P.; Kratochwil, C.; Brunegraf, A.; Gosewisch, A.; Gildehaus, F.J.; Tritschler, S.; Stief, C.G.; Kopka, K.; Haberkorn, U.; et al. Dosimetry for ${ }^{177}$ Lu-DKFZ-PSMA-617: A new radiopharmaceutical for the treatment of metastatic prostate cancer. Eur. J. Nucl. Med. Mol. Imaging 2016, 43, 42-51. [CrossRef] [PubMed]

13. Violet, J.; Jackson, P.; Ferdinandus, J.; Sandhu, S.; Akhurst, T.; Iravani, A.; Kong, G.; Kumar, A.R.; Thang, S.P.; Eu, P.; et al. Dosimetry of ${ }^{177}$ Lu-PSMA-617 in metastatic castration-resistant prostate cancer: Correlations between pretherapeutic imaging and whole-body tumor dosimetry with treatment outcomes. J. Nucl. Med. 2019, 60, 517-523. [CrossRef]

14. DGN-Geschäftsstelle. RLT mit Lutetium-177 Markiertem PSMA (Prostata Spezifisches Membranantigen) Antagonist (Lu-177 DKFZ-PSMA-617). Available online: https: / www.google.com.hk/url?sa=t\&rct=j\&q=\&esrc=s\&source=web\&cd=\&cad=rja\& uact=8\&ved=2ahUKEwj0sNSi5oTvAhUCHqYKHda-CVsQFjAAegQIAxAD\&url=https\%3A\%2F\%2Fwww.nuklearmedizin. de\%2Fdocs\%2FLu-177-PSMA_160224.pdf\&usg=AOvVaw0hdulCzjGXOZo3dk3Gwgnj (accessed on 20 December 2020).

15. Fendler, W.P.R.; Reinhardt, S.; Ilhan, H.; Delker, A.; Böning, G.; Gildehaus, F.J; Stief, C.; Bartenstein, P.; Gratzke, C.; Lehner, S.; et al. Preliminary experience with dosimetry, response and patient reported outcome after ${ }^{177}$ Lu-PSMA-617 therapy for metastatic castration-resistant prostate cancer. Oncotarget 2017, 8, 3581-3590. [CrossRef] [PubMed]

16. Weineisen, M.; Simecek, J.; Margret, S.; Markus, S.; Hans-Jürgen, W. Synthesis and preclinical evaluation of DOTAGAconjugated PSMA ligands for functional imaging and endoradiotherapy of prostate cancer. EJNMMI Res. 2014, 4, 63. [CrossRef]

17. Giesel, F.L.; Hadaschik, B.; Cardinale, J.; Radtke, J.; Vinsensia, M.; Lehnert, W.; Kesch, C.; Tolstov, Y.; Singer, S.; Grabe, N.; et al. F-18 labelled PSMA-1007: Biodistribution, radiation dosimetry and histopathological validation of tumor lesions in prostate cancer patients. Eur. J. Nucl. Med. Mol. Imaging 2017, 44, 678-688. [CrossRef]

18. Collarino, A.; Pereira Arias-Bouda, L.M.; Valdés Olmos, R.A.; van der Tol, P.; Dibbets-Schneider, P.; de Geus-Oei, L.-F.; van Velden, F.H.P. Experimental validation of absolute SPECT/CT quantification for response monitoring in breast cancer. Med. Phys. 2018, 45, $2143-2153$. [CrossRef] [PubMed] 
19. Violet, J.; Sandhu, S.; Iravani, A.; Ferdinandus, J.; Thang, S.P.; Kong, G.; Kumar, A.R.; Akhurst, T.; Pattison, D.A.; Beaulieu, A.; et al. Long-term follow-up and outcomes of retreatment in an expanded 50-patient single-center phase II prospective trial of. J. Nucl. Med. 2020, 61, 857-865. [CrossRef]

20. Maffey-Steffan, J.; Scarpa, L.; Svirydenka, A.; Nilica, B.; Nilica, B.; Mair, C.; Buxbaum, S.; Bektic, J.; von Guggenberg, E.; Uprimny, C.; et al. The $(68) \mathrm{Ga} /\left({ }^{177}\right)$ Lu-theragnostic concept in PSMA-targeting of metastatic castration-resistant prostate cancer: Impact of post-therapeutic whole-body scintigraphy in the follow-up. Eur. J. Nucl. Med. Mol. Imaging 2020, 47, 695-712. [CrossRef]

21. Grubmüller, B.; Senn, D.; Kramer, G.; Baltzer, P.; D'Andrea, D.; Grubmüller, K.H.; Mitterhauser, M.; Eidherr, H.; Haug, A.R.; Wadsak, W.; et al. Response assessment using (68)Ga-PSMA ligand PET in patients undergoing ( ${ }^{177}$ )Lu-PSMA radioligand therapy for metastatic castration-resistant prostate cancer. Eur. J. Nucl. Med. Mol. Imaging 2019, 46, 1063-1072. [CrossRef]

22. Joo Hyun, O.; Martin, A.L.; Richard, L.W. Practical PERCIST: A simplified guide to PET response criteria in solid tumors 1.0. Radiology 2016, 280, 576-584. [CrossRef]

23. Eisenhauer, E.A.; Therasse, P.; Bogaerts, J.; Schwartz, L.H.; Schwartz, L.; Sargent, D.; Ford, R.; Dancey, J.; Arbuck, S.; Gwyther, S.; et al. New response evaluation criteria in solid tumours: Revised RECIST guideline (version 1.1). Eur. J. Cancer 2009, 45, $228-247$. [CrossRef]

24. Seitz, A.K.; Rauscher, I.; Haller, B.; Krönke, M.; Luther, S.; Heck, M.M.; Horn, T.; Gschwend, J.E.; Schwaiger, M.; Eiber, M.; et al. Preliminary results on response assessment using 68Ga-HBED-CC-PSMA PET/CT in patients with metastatic prostate cancer undergoing docetaxel chemotherapy. Eur. J. Nucl. Med. Mol. Imaging 2018, 45, 602-612. [CrossRef]

25. Powers, E.; Karachaliou, G.S.; Kao, C.; Harrison, M.R.; Hoimes, C.J.; George, D.J.; Armstrong, A.J.; Zhang, T. Novel therapies are changing treatment paradigms in metastatic prostate cancer. J. Hematol. Oncol. 2020, 13, 144. [CrossRef]

26. Kluetz, P.G.; Pierce, W.; Maher, V.E.; Zhang, H.; Tang, S.; Song, P.; Liu, Q.; Haber, M.T.; Leutzinger, E.E.; Al-Hakim, A.; et al. Radium Ra 223 dichloride injection: U.S. Food and Drug Administration drug approval summary. Clin. Cancer Res. 2014, 20, 9-14. [CrossRef]

27. Eberlein, U.; Cremonesi, M.; Lassmann, M. Individualized dosimetry for theranostics: Necessary, nice to have, or counterproductive? J. Nucl. Med. 2017, 58, 97S-103S. [CrossRef]

28. Pfestroff, A.; Luster, M.; Jilg, C.A.; Olbert, P.J.; Ohlmann, C.H.; Lassmann, M.; Maecke, H.R.; Ezziddin, S.; Bodei, L.; Radionuclide Therapy Committee of the European Association of Nuclear Medicine. Current status and future perspectives of PSMA-targeted therapy in Europe: Opportunity knocks. Eur. J. Nucl. Med. Mol. Imaging 2015, 42, 1971-1975. [CrossRef]

29. Zacho, H.D.; Nielsen, J.B.; Haberkorn, U.; Stenholt, L.; Petersen, L.J. (68) Ga-PSMA PET/CT for the detection of bone metastases in prostate cancer: A systematic review of the published literature. Clin. Physiol. Funct. Imaging 2017. [CrossRef]

30. Gupta, M.; Choudhury, P.S.; Rawal, S.; Goel, H.C.; Rao, S.A. Evaluation of RECIST, PERCIST, EORTC, and MDA criteria for assessing treatment response with Ga68-PSMA PET-CT in metastatic prostate cancer patient with biochemical progression: A comparative study. Nucl. Med. Mol. Imaging 2018, 52, 420-429. [CrossRef]

31. Fanti, S.; Goffin, K.; Hadaschik, B.A.; Herrmann, K.; Maurer, T.; MacLennan, S.; Oprea-Lager, D.E.; Oyen, W.J.; Rouviere, O.; Mottet, N.; et al. Consensus statements on PSMA PET/CT response assessment criteria in prostate cancer. Eur. J. Nucl. Med. Mol. Imaging 2021, 48, 469-476. [CrossRef]

32. Heinzel, A.; Boghos, D.; Mottaghy, F.M.; Gaertner, F.; Essler, M.; von Mallek, D.; Ahmadzadehfar, H. (68)Ga-PSMA PET/CT for monitoring response to $\left({ }^{177}\right)$ Lu-PSMA-617 radioligand therapy in patients with metastatic castration-resistant prostate cancer. Eur. J. Nucl. Med. Mol. Imaging 2019, 46, 1054-1062. [CrossRef]

33. Sartor, O.; Coleman, R.E.; Nilsson, S.; Heinrich, D.; Helle, S.I.; O'Sullivan, J.M.; Vogelzang, N.J.; Bruland, O.; Kobina, S.; Wilhelm, S.; et al. An exploratory analysis of alkaline phosphatase, lactate dehydrogenase, and prostate-specific antigen dynamics in the phase 3 ALSYMPCA trial with radium-223. Ann. Oncol. 2017, 28, 1090-1097. [CrossRef] [PubMed]

34. Derlin, T.; Schmuck, S. [ $\left.\left({ }^{177}\right) \mathrm{Lu}\right]$-PSMA-617 radionuclide therapy in patients with metastatic castration-resistant prostate cancer. Lancet Oncol. 2018, 19, e372. [CrossRef]

35. Rathke, H.; Giesel, F.L.; Flechsig, P.; Kopka, K.; Mier, W.; Hohenfellner, M.; Haberkorn, U.; Kratochwil, C. Repeated ( ${ }^{177}$ )Lu-labeled PSMA-617 radioligand therapy using treatment activities of up to $9.3 \mathrm{GBq}$. J. Nucl. Med. 2018, 59, 459-465. [CrossRef]

36. Finocchiaro, D.; Gear, J.I.; Fioroni, F.; Flux, G.D.; Murray, I.; Castellani, G.; Versari, A.; Iori, M.; Grassi, E. Uncertainty analysis of tumour absorbed dose calculations in molecular radiotherapy. EJNMMI Phys. 2020, 7, 63. [CrossRef] 